Adapting patient consent in response

\title{
to COVID-19
}

\section{Jane Merivale}

Senior dento-legal advisor, BDA Indemnity

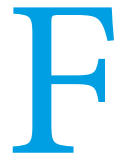

ollowing any significant and unpleasant event there are likely to be consequences - the so-called aftermath - and the COVID-19 pandemic certainly qualifies as just such an event. We are all too well aware that COVID-19 is a virus that society and the dental profession will have to live with for the foreseeable future, accommodating within our psyche the possibility of additional 'spikes' and local lockdowns. The prevailing uncertainty makes it difficult to know if the dental profession is now addressing the aftermath or moving into a new existence requiring agile and scaleable resourcing which will also impact on our businesses, personal health and wellbeing, and patient care.

Whilst the public has vocalised its appreciation of healthcare professionals, notably with the weekly 'Clap for Carers', experience tells us that the tide can turn. Frustrations are tolerated for only so long and as people vie for appointments there is an associated fear amongst dentists that complaints will arise accompanied by the unwanted spectre of litigation.
For example, could a patient claim that they acquired COVID-19 during a visit to the dentist? BDA Indemnity has regularly been asked if there is any consent form a patient could sign that would act as a 'legal flak jacket' and effectively protect the dentist from litigation.

There is no simple solution and the time-honoured necessity of following the available guidance and keeping meticulous clinical and administrative records still applies. Central to this will be effective communication with patients and care during the consent process.

BDA Indemnity is carefully curating a timeline of guidance as it evolves during the pandemic so that in the event of a dentist being challenged about the care they provided on a specific date, the practice's adherence to the current Standard Operating Procedure (SOP) and guidance, that would have been in place at the time can be readily identified and deployed in their defence. It is therefore imperative that all documentation and compliance within the practice, training, team meetings and practical measures adopted within the practice can be demonstrated 
in response to any challenge. For example, photographs of the premises, daily screening records of the staff and risk assessments would be invaluable.

With so much to think about, a frequent question that arises is whether templates can be used to facilitate record keeping. It is important to be familiar with the prevailing record keeping guidance ${ }^{1}$ to make sure you are following good practice; no template can cover all eventualities or circumstances but it is a useful aide memoire of basic details that can be augmented. This is particularly the case in relation to the PPE used by team members when treating patients e.g. FFP2 masks (or other models), gowns as well as other mitigating measures such as rubber dam, high volume evacuation and pretreatment rinses.

Dentists always need to exercise their clinical judgement when making notes that capture the context and the patient's particular circumstances. ${ }^{2}$ As with consent, and the changes adopted as a result of the Montgomery case, records and consent need to be tailored to the individual patient and therefore generic consent forms and record keeping templates can only ever serve as a guide.

There is no substitute for good communication, and it is important that what is written in the records actually took place; the patient may argue that there was no such dialogue and no such agreement to proceed on those terms.

In order to be valid, the consent process needs to be tailored to each patient and their particular circumstances, taking into account what matters most to them. Essentially, this summarises the legal precedent created by the Montgomery case in 2015.

Hence your records are the only sure method of demonstrating the consent process which will inevitably evolve over the period you are treating the patient; remembering also that the patient can withdraw their consent at any point.

When providing dental treatment, it is important that every patient fully understands any dental treatment that is proposed in order to make an informed decision about how they would like to proceed. There are several aspects to consider when discussing treatment with the patient in order to ensure that their consent is valid, as shown in Box 1 .
Having gone through these stages and fully documented them within the record, the patient can be deemed to have given their valid consent based on the information received and their confirmation that they have understood what the clinician has told them. You can be confident that your records of the consent process are adequate provided they demonstrate the three underlying pillars of consent:

1. The patient has been given sufficient information

2. They have been able to understand it and have had sufficient time and opportunity to weigh up their options

3. They are able to articulate their decision to you as the treating dentist.

\section{'There is no substitute for good communication, and it is important that what is written in the records actually took place; the patient may argue that there was no such dialogue and no such agreement to proceed on those terms.'}

A signature to this effect is not required; but your clinical notes are crucial such that the detail they contain as well as their veracity is most important.

Due to the additional challenges of COVID-19 you currently need to document the steps you have taken to comply with the latest guidance and to ensure the patient understands these and confirms their willingness to undergo treatment in this context. There may be limits on the treatments you can offer depending on national guidance as well your own local practice circumstances regarding the availability of the necessary level of PPE or scheduling of appointments around aerosol generating procedures (AGP) and non-AGP clinics. Remember that whilst we have become fluent in new words and acronyms (eg.'PPE', 'SOP' and 'AGP') there needs to be clear communication with the patient and an explanation of how they might apply to their proposed episode of treatment.
Box 1 How to ensure patient consent is valid

$\rightarrow$ The patient is made fully aware of their diagnosis in words they can understand

$\rightarrow$ The patient has been enabled to understand the treatment options available to treat the condition which has been diagnosed (which includes no treatment, and the associated implications)

$\rightarrow$ The nature of the treatment being proposed (i.e. what the treatment entails, what it will be like)

$\rightarrow$ The risks and benefits of the various treatment options

$\rightarrow$ The patient has been invited to ask any questions and the answers have been given and understood

$\rightarrow$ The final decision is made by that particular patient

Finally, if you are challenged at a later date by a patient, the quality of your defence will be based on the records you have kept, your ability to demonstrate compliance with current guidance and the effectiveness of your communication with your patients and the understanding they have gained as a consequence.

The dental advisors working for BDA Indemnity understand that there are a number of adjustments that the dental team will need to make as the pandemic evolves which may seem cumbersome when compared with previously accepted treatment protocols and can increase any existing anxieties that COVID-19 might have created for members of the dental team.

Members can find the BDA's latest advice relating to COVID-19 by visiting https:// www.bda.org/advice/Coronavirus •

\section{References}

1. FGDP(UK). Clinical Examination and Record Keeping. Available online at: https://www. fgdp.org.uk/guidance-standards/clinicalexamination-and-record-keeping-0

(Accessed July 2020).

2. Montgomery v Lanarkshire (2015) UKSC 11

https://doi.org/10.1038/s41404-020-0470-0 